\title{
Planning of Shelf Operation in Dysplastic Hip by CT and MRI Based Finite Element Contact Analysis*
}

\author{
Yoon Hyuk KIM**, Won Man PARK ${ }^{* *}$, Kyungsoo KIM**, \\ Won Joon $\mathrm{YOO}^{* * * *}$, Tae Joon $\mathrm{CHO}^{* * * *}$ and In Ho $\mathrm{CHOI}^{* * * *}$
}

\begin{abstract}
Finite element contact analyses of dysplastic hip joints were performed based on CT and MR images as a surgical planning tool of the shelf operation. The 3-D cartilage thickness was approximated using MRI, and the joint contact force was calculated from a 3-D expansion of the Ninomiya's method. After surgical planning, the anatomical parameters including the CE angle, the $\mathrm{AC}$ angle, the sharp angle and the spheric sector angle were improved to normal hips. The mechanical parameters including the maximum contact pressure, the contact area and the quality of contact pressure distribution also were improved. The present models and the results can be used as a computer simulation tool for optimal pre-operative planning of the shelf operation in hip dysplasia.
\end{abstract}

Key Words: Hip Dysplasia, Shelf Operation, Contact Pressure, Finite Element Analysis, Surgical Planning

\section{Introduction}

Developmental dysplasia of the hip is a spectrum of disorders affecting the proximal femur and acetabulum that are subluxatable, dislocatable, and dislocated ${ }^{(1)-(3)}$. This dysplastic hip is the result of a disruption in the normal relationship between the acetabulum and the femoral head. If dysplastic hip is not treated promptly, it could generate osteoarthritis, severe pain, abnormal gait and unequal leg-length ${ }^{(1)-(3)}$. The major biomechanical changes of dysplastic hip are the contact pressure and contact area, and the anatomical configuration.

Several surgical options, such as the Salter osteotomy, the spherical acetabular osteotomy, the Chiari ostoetomy, and the shelf operation, have been applied to treat hip dysplasia $^{(1)-(3)}$. These surgical approaches mainly change the relative rotation and location between the acetabulum and the femoral head, and increase the contact coverage, so that the contact pressure of the hip joint are decreased and

\footnotetext{
* Received 2nd June, 2005 (No. 05-5058)

** School of Advanced Technology, Kyung Hee University, 1 Sochen-ni, Kihung-eop, Yongin-shi, Kyongki-do 449701, Korea. E-mail: yoonhkim@khu.ac.kr

*** Department of Mathematical Sciences, Seoul National University, San56-1, Shinrim-dong, Kwanak-gu, Seoul 151-747, Korea

**** College of Medicine, Seoul National University, 28 Yongon-dong, Chongno-gu, Seoul 110-799, Korea
}

redistributed. The shelf operation has been a major surgical option in children over 8 years old. This operation provides adequate support to the subluxated femoral head and ensures a stable mobile joint capable of standing up to reasonable used for many years ${ }^{(4)}$. The advantages of this operation are the biomechanical elimination of the shear force in subluxation, increase of femoral head coverage with coax magna, and no change in the femoral neck-shaft angle.

In conventional pre-operative planning of hip dysplasia, the anatomical angles and lines of the hip and the femoral head are measured using a frontal radiographic image $^{(5),(6)}$. However, this approach is indirect since we assume the normal anatomical configuration would provide the normal range of contact pressure distribution. Therefore, the direct estimation of the contact pressure in the hip joint can provide more direct information in surgical planning.

Finite element method has been widely used to estimate the contact pressure of the hip joint since it is very difficult to measure the contact pressure experimentally. However, to our knowledge, most of the contact pressure analyses are 2-D in frontal plane or 3-D based on the assumption that the contact surface is sphere ${ }^{(6)-(10)}$. In addition, previous finite element analysis studies have assumed that cartilage thickness is constant along the entire hip joint and the joint contact force is determined from 2-D based the Ninomiya's method ${ }^{(6)-(9)}$. 
In this study, the finite element analyses of the contact pressures in the pre-operative and post-operative dysplastic hip joints of two patients were performed based on CT and MR images. The finite element models of the bones were developed based on CT slices and the cartilage models were developed based on MRI. A new 3-D expansion of the Ninomiya's method was suggested to determine the joint contact force and abductor force in the hip from the 3-D FE model. Contact pressure distributions and the contact area were determined as well as anatomical parameters of the hip models. The present study was intended to use as a planning guide of the shelf operation in dysplastic hip.

\section{Materials and Methods}

\subsection{CT and MRI based finite element modeling}

Two patients with dysplastic left hips whose weights have 26 and $33 \mathrm{~kg}$ were selected. $2 \mathrm{~mm}$ slices of CT images from the proximal femur to the pelvis were reconstructed to solid models using 3D-Doctor (Able Software 3d-doctor, U.S.A.) for boundary edge detection of each slice and Rapidform (Inus Tech. Inc., Korea) for converting the images to IGES type model ${ }^{(11),(12)}$. From the solid models, finite element meshes were developed with linear tetrahedral elements using Femap ver. 8.2 (Fig. 1; UGS Inc., TX, U.S.A.). The post-operative finite element models of the shelf operation were developed based on the orthopedic clinical guideline (Fig. 2) ${ }^{(13)}$. First, the outer cortex of the ilium exposed sub-periosteally down to the joint capsule. Then bone cutting was introduced along the joint capsule into the rim of the acetabulum, and laterally distracted from $17.4 \mathrm{~mm}$ for patient 1 and $19.6 \mathrm{~mm}$ for patient 2 , and this distraction length was represented as $l_{d}$. Finally

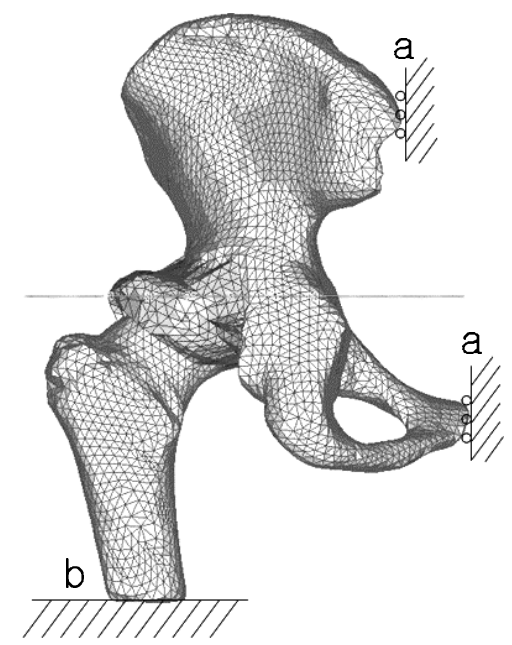

Fig. 1 The pre-operative finite element model of dysplastic hip reconstructed from CT and MRI. The model covers the proximal femur, the acetabulum and the pelvis. For the boundary conditions, the distal femur is fixed and the pelvis is allowed to displace vertically bone grafts were inserted to the free space and the lateral edge to cover the contact surface.

The cartilage thickness distribution of each model was measured using MR images with $512 \times 512$ matrix, which is about $0.51 \mathrm{~mm}$ of spatial resolution (Magnetom Vision Plus, 1.5 Tessler, Siemens, Germany) (Fig. 3). Due to the resolution limit of the MR images, we averaged the measured cartilage thickness by three different clinicians for three times each. Since the cartilage thickness is threedimensional, we followed the Nishii's method ${ }^{(14)}$. In anteroposterior and lateral MR images, the thicknesses of the acetabular and the femoral cartilages were measured along the radial directions, $0^{\circ}, \pm 20^{\circ}$, and $\pm 40^{\circ}$, from the center of the femoral head. Next, both lateral and medial ends of the cartilage thicknesses were measured. Then, a 3-D surface approximation of the cartilage thickness was reconstructed using Femap ver. 8.2 (UGS Inc., TX, U.S.A.). Before measuring the cartilage thicknesses from MR images, we have matched those MR images with frontal and sagittal images of the FE model reconstructed from CT slices in order to have same scale of the two images.

In this study, the materials were assumed as linear elastic and frictional contact analyses were performed. The material properties of the cortical bone and cartilage were used from the literatures as $E_{\text {cortical }}=17 \mathrm{GPa}$, $E_{\text {cartilage }}=15 \mathrm{MPa}, v_{\text {cortical }}=0.3, v_{\text {cartilage }}=0.45$, and $\mu=$ $0.02^{(9)}$. For boundary conditions of the analyses, the distal femur was fixed and the nodes of the pelvis were allowed to move vertical only (Fig. 1$)^{(9)}$. The contact analyses were performed using ABAQUS/Standard 6.3 (ABAQUS Inc., RI, U.S.A.).

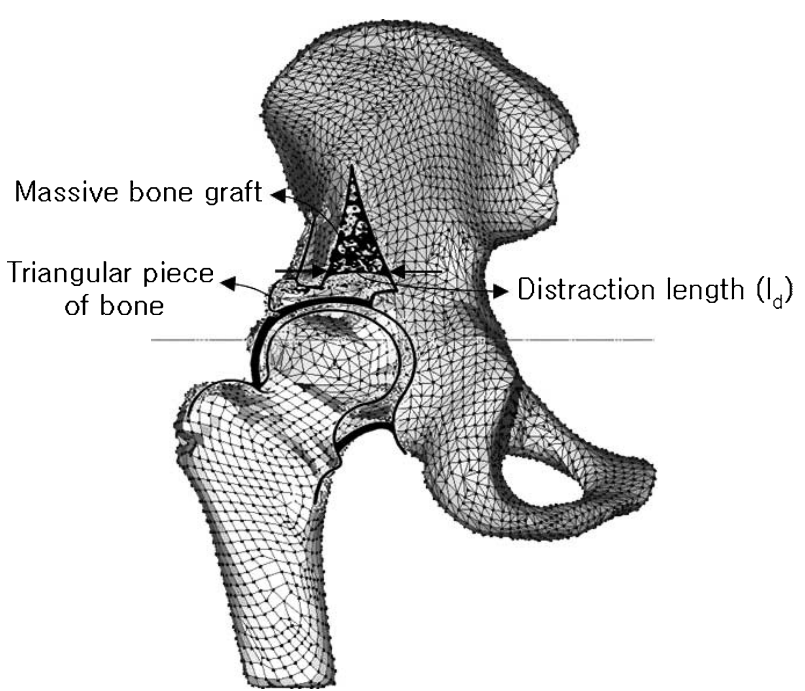

Fig. 2 The post-operative finite element model of dysplastic hip following the shelf operation approach. The outer cortex of the ilium exposed sub-periosteally down to the joint capsule. Then bone cutting was introduced along the joint capsule into the rim of the acetabulum, and laterally distracted from $17 \mathrm{~mm}$ to $20 \mathrm{~mm}\left(\boldsymbol{l}_{d}\right)$ 


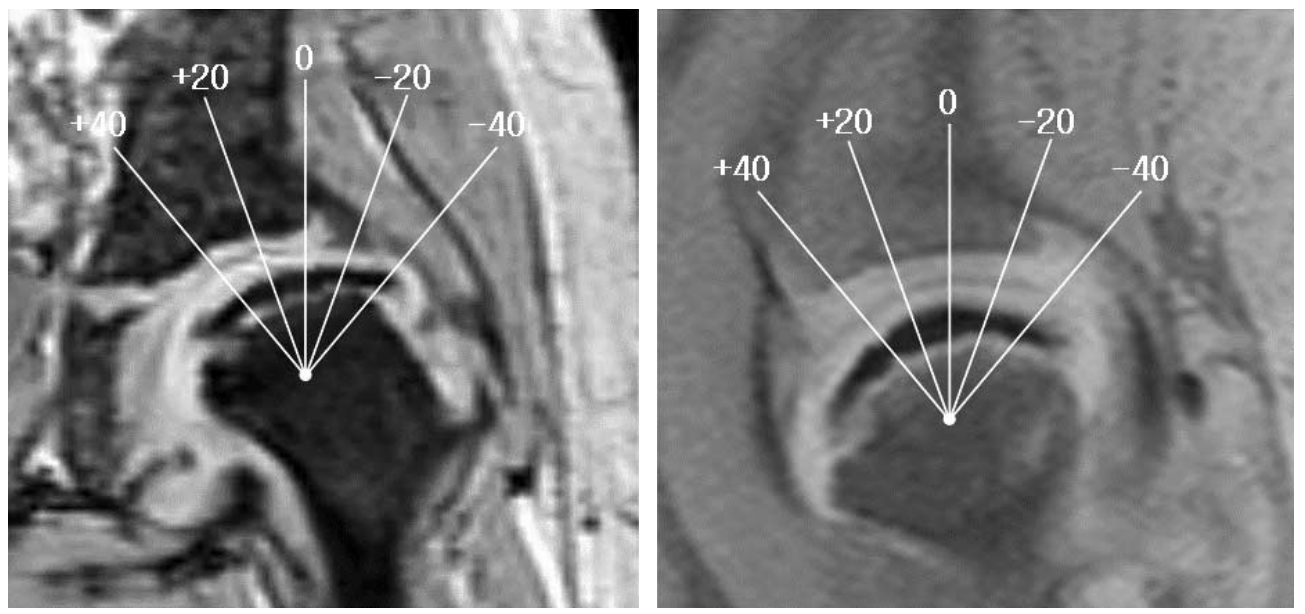

Fig. 3 The MR images of a left dysplastic hip in 8-year-old patient. Acetabular and femoral cartilages were measured along the radial directions from the femoral center. (a) Midfrontal MR image. (b) Mid-sagittal MR image

\subsection{3-D determination of resultant joint force}

To determine the resultant joint force and abductor force in three-dimension, we expanded the Ninomiya's method (Fig. 4) ${ }^{(5),(6)}$. In this method, the abductor muscle direction could be estimated from the frontal and the sagittal views of the finite element model reconstructed from CT. The most lateral point on the ilium (point 2) and the nearest point on the inner edge of the ilium to point 3 were digitized. Next, point 4 was defined as one third of the distance from point 2 to point 3 . Then, the line between the most lateral point of the greater trochanter (point 1) and point 4 was defined as the direction of the abductor muscle force. In this study, single leg standing posture was assumed and five-sixth of the body weight was applied through the fifth lumbar vertebra in a vertical direction. Then, the resultant joint force and the abductor force were calculated from the 3-D static equilibrium at the hip joint center as follows:

$$
\begin{aligned}
& \tan \alpha=\frac{F_{x}}{F_{z}}, \quad \tan \gamma=\frac{F_{y}}{F_{z}} \\
& \sqrt{F_{x}^{2}+F_{z}^{2}}(d-e)(\cos \alpha-\sin \alpha \cdot \tan \alpha)=W \cdot e, \\
& F_{x}=\frac{\tan \alpha}{1-\tan \alpha \cdot \tan \beta} \cdot \frac{e}{d-e} \cdot W, \\
& F_{y}=\frac{\tan \gamma}{1-\tan \alpha \cdot \tan \beta} \cdot \frac{e}{d-e} \cdot W, \\
& F_{z}=\frac{1}{1-\tan \alpha \cdot \tan \beta} \cdot \frac{e}{d-e} \cdot W, \\
& R_{x}=-F_{x}, \quad R_{y}=-F_{y}, \quad R_{z}=-F_{z}-W,
\end{aligned}
$$

where $\boldsymbol{F}=\left(F_{x}, F_{y}, F_{z},\right)$ is the abductor force, $W=5 / 6$ of body weight, $\boldsymbol{R}=\left(R_{x}, R_{y}, R_{z},\right)$ is the resultant joint force. $\alpha$ and $\gamma$ are the frontal and sagittal angles of the abductor force, $\beta$ is the angle between the line connecting the hip joint center and point 1 and the horizontal. Line $d$ is the horizontal distance between the hip joint center and body
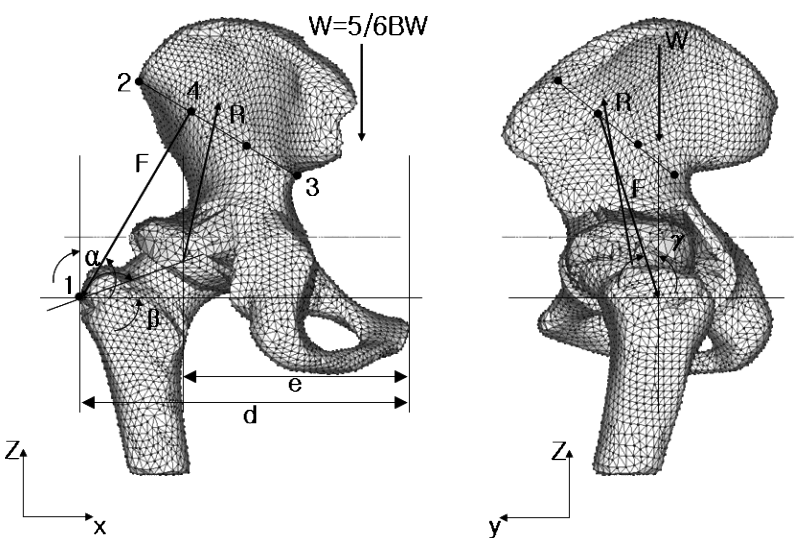

Fig. 4 A 3-D expansion of the Ninomiya's method to calculate the resultant joint force $(\boldsymbol{R})$ and the abductor force $(\boldsymbol{F})$. The abductor force is assumed to be in the direction of line $1-4$ and point 4 is $1 / 3$ the distance of line $2-$ 3. The joint force is calculated from the $3-\mathrm{D}$ static equilibrium equations at the hip joint center in one-leg standing position

weight line, and line $e$ is the horizontal distance between the hip joint center and point 1 .

\subsection{Determination of anatomical parameters}

The anatomical parameters for the surgical planning of the shelf operation were defined using the landmarks $(\text { Fig. } 5)^{(6)}$. The CE angle was determined by the femoral head center and point 5 , the sharp angle by points 5 and 6 , the acetabular cartilage (AC) angle by the horizontal line and points 5 and 7 , and the spheric sector angle by the femoral center and points 5 and 7 , respectively.

\subsection{Evaluation of surgical planning of the shelf op- eration}

For the evaluation of the surgical planning of the shelf operation, the four anatomical parameters described in the paper were measured from the pre-operative and the postoperative finite element models, and the post-operative X- 


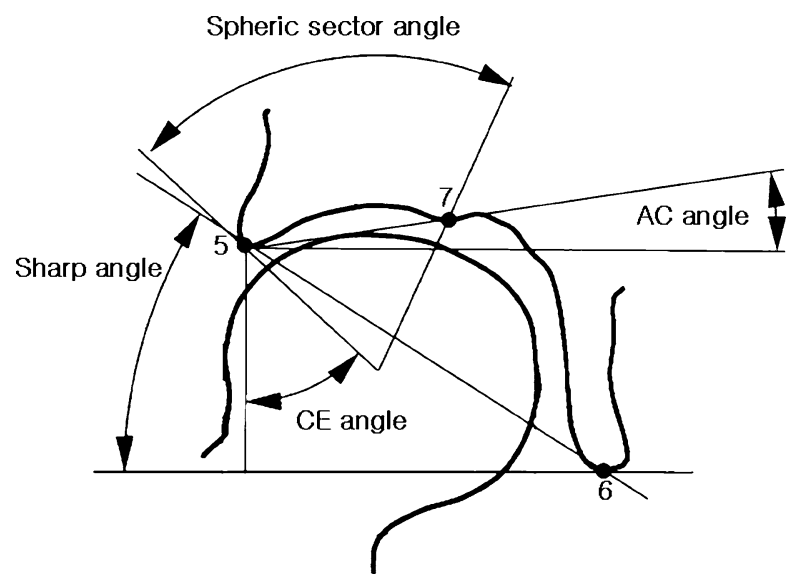

Fig. 5 Four anatomical parameters used in the planning guideline of the shelf surgery ${ }^{(6)}$

ray images. In addition the mechanical parameters, which are the maximum contact pressure, the contact area and the ratio of the contact area over $60 \%$ of the maximum contact pressure $\left(\mathrm{CA}_{60}\right)$, were calculated from the contact analysis.

\section{Results}

The acetabular and femoral cartilage thicknesses of the two children dysplastic hips were much higher and irregular compared with the averaged those values in adult normal and dysplastic hips, $1.34 \pm 0.15 \mathrm{~mm}$ and $1.77 \pm$ $0.31 \mathrm{~mm}$, respectively (Table 1$)^{(14)}$.

The anatomical parameters of the post-operative models were shifted to the normal populations compared with those in the pre-operative models (Table 2$)^{(6)}$. The average $\mathrm{CE}$ angle was increased from $6.1^{\circ}$ to $40^{\circ}$ close to the normal value in adults, $28^{\circ}$. The average spheric sector angle was increased from $50^{\circ}$ to $82^{\circ}$ close to the normal value in adults, $68^{\circ}$. The average sharp angle in the post-operative models was $43^{\circ}$ close to $39^{\circ}$, the normal value in adults while that in the pre-operative models was $30^{\circ}$. From the comparison of the anatomical parameters, the surgical planning of the shelf operation could be clinically feasible. In addition, the anatomical parameters of the post-operative models were close to those measured in plane X-rays post-operatively (Table 2).

The mechanical parameters, which include the maximum contact pressure, the contact area, and the ratio of the contact area over $60 \%$ of the maximum contact pressure to the total contact area, $\mathrm{CA}_{60} /$ Contact area were, calculated from the finite element contact analyses of preoperative and post-operative hip models (Table 2). The maximum contact pressures were decreased from $2.3 \mathrm{MPa}$ to $1.6 \mathrm{MPa}$ in patient 1 and from $6.0 \mathrm{MPa}$ to $4.5 \mathrm{MPa}$ in patient 2 . The contact areas were increased by $22 \%$ in patient 1 and by $23 \%$ in patient 2 . The ratios of the contact area over $60 \%$ of the maximum contact pressure were dramatically decreased from $33 \%$ to $8 \%$ in patient 1 and $35 \%$
Table 1 The thicknesses of the acetabular and femoral cartilage in mid-frontal and mid-sagittal plane

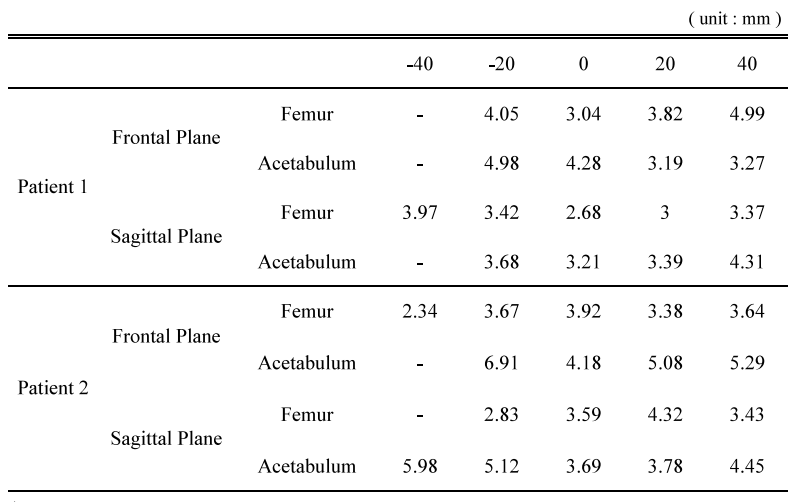

* Note : The averaged adult acetabular cartilage thicknesses in normal and dysplastic hips were $1.34 \pm 0.15 \mathrm{~mm}$ and $1.77 \pm 0.31 \mathrm{~mm}$, respectively [14].

Table 2 The anatomical and mechanical parameters of the pre-operative and the post-operative model with the measured anatomical parameters from post-operative X-ray images for the two patients

\begin{tabular}{|c|c|c|c|c|c|}
\hline & & & $\begin{array}{l}\text { Pre-Op. } \\
\text { (FEM) }\end{array}$ & $\begin{array}{c}\text { Post-Op. } \\
\text { (FEM) }\end{array}$ & $\begin{array}{l}\text { Post-Op. } \\
\text { (X-Ray) }\end{array}$ \\
\hline \multirow{8}{*}{ Patient 1} & \multirow{4}{*}{$\begin{array}{l}\text { Anatomic } \\
\text { parameter }\end{array}$} & $\mathrm{CE}$ angle $\left({ }^{\circ}\right)$ & 3.4 & 44.2 & 39.5 \\
\hline & & AC angle $\left({ }^{\circ}\right)$ & -11.3 & -7.2 & -7.6 \\
\hline & & Spheric sector angle $\left({ }^{\circ}\right)$ & 47.0 & 84.3 & 74.0 \\
\hline & & Sharp angle ( ${ }^{\circ}$ ) & 42.5 & 32.5 & 39.5 \\
\hline & \multirow{4}{*}{$\begin{array}{c}\text { Mechanical } \\
\text { Parameter }\end{array}$} & Maximum Von mises stress ( $\mathrm{MPa}$ ) & 0.55 & 0.38 & \\
\hline & & Maximum Contact Pressure (MPa) & 2.30 & 1.60 & \\
\hline & & Contact Area $\left(\mathrm{mm}^{2}\right)$ & 1092.8 & 1399.7 & \\
\hline & & $\mathrm{CA}_{60} /$ Contact Area (\%) & 32.6 & 8.4 & \\
\hline \multirow{8}{*}{ Patient 2} & \multirow{4}{*}{$\begin{array}{l}\text { Anatomic } \\
\text { parameter }\end{array}$} & $\mathrm{CE}$ angle $\left({ }^{\circ}\right)$ & 8.7 & 37.4 & 33.0 \\
\hline & & AC angle $\left({ }^{\circ}\right)$ & -0.4 & 2.3 & 1.5 \\
\hline & & Spheric sector angle $\left({ }^{\circ}\right)$ & 52.2 & 80.7 & 71.0 \\
\hline & & Sharp angle $\left({ }^{\circ}\right)$ & 44.6 & 28.5 & 32.0 \\
\hline & \multirow{4}{*}{$\begin{array}{c}\text { Mechanical } \\
\text { Parameter }\end{array}$} & Maximum Von mises stress (MPa) & 1.39 & 1.14 & \\
\hline & & Maximum Contact Pressure (MPa) & 6.05 & 4.51 & \\
\hline & & Contact Area $\left(\mathrm{mm}^{2}\right)$ & 1536.1 & 1994.1 & \\
\hline & & $\mathrm{CA}_{60} /$ Contact Area (\%) & 34.6 & 15.9 & \\
\hline
\end{tabular}

* Note : $\mathrm{CA}_{60}$ is the area over $60 \%$ of the maximum contact pressure.

to $16 \%$ in patient 2 . Figure 6 showed that the contact areas were increased and high contact pressures were decreased in the post-operative models of the two patients.

\section{Discussion}

In the surgical planning of hip dysplasia, there are several anatomical parameters used to describe the deformities of the hip joints. These parameters varied between genders, ages and the source of the diseases ${ }^{(6)}$. Especially in the hip dysplasia of children, the anatomical parameters are much different from those in adults, and this deformity of the dysplastic hip needs precise 3-D surgical planning. The change of the anatomical parameters due to hip dysplasia causes the change of the contact pressure distribution and the contact area, and this hip deformity subsequently induces osteoarthritis. So, it is important to estimate the contact pressures directly to plan the shelf surgery for hip dysplasia. To our knowledge, it is the first 
( unit : MPa)

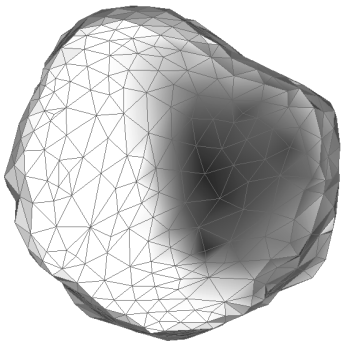

(a)

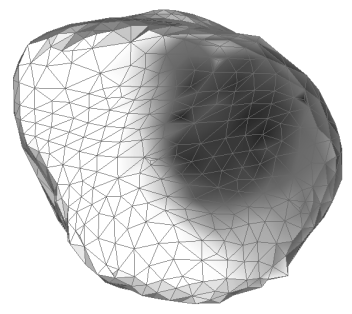

(c)
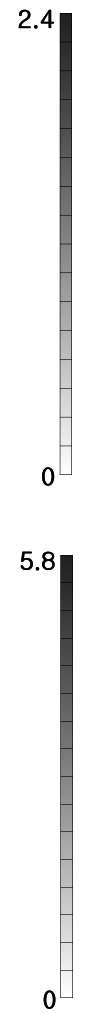

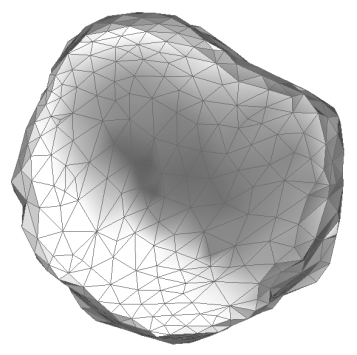

(b)

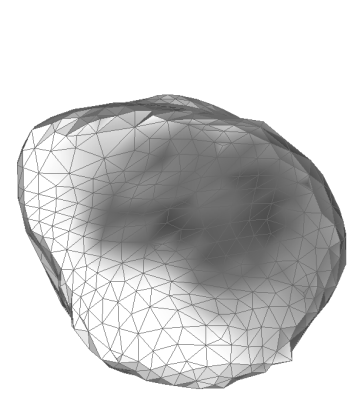

(d)
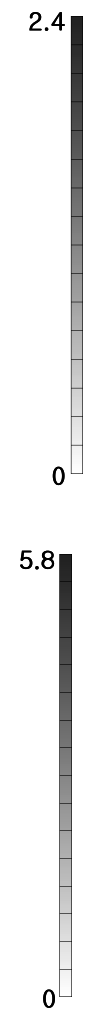

Fig. 6 The contact pressure distribution on the femoral cartilage surface. (a) pre-operative FE model of patient 1. (b) post-operative FE model of patient 1. (c) pre-operative FE model of patient 2. (d) post-operative FE model of patient 2

study to estimate the contact pressures of dysplastic hip of children.

In the finite element contact analysis for the surgical planning of the shelf operation, most of the deformations and pressures occur in the cartilage, thus the reconstruction of the acetabular and femoral cartilages in the model is important to estimate the accurate contact pressures. However, previous studies have assumed that cartilage thickness is either constant, or equally distributed to both acetabular and femoral sides. In children case, the cartilage thicknesses in acetabular and fermoral sides are much different. Since cartilage tissue can not be measured from plane X-ray, MRI is necessary to provide the direct visualization of the articular cartilage thickness. However, this is not convenient and expensive to take a lot of MR images to reconstruct the articular cartilage. In this study, we could use frontal and sagittal MR images to measure the cartilage thickness in radial direction, and reconstruct the 3-D contact surface approximately using these measured thickness values. This simple method is an approximate one, but it could minimize the use of MRI to obtain the cartilage thicknesses in acetabular and femoral sides. From the results, the cartilage thicknesses in the dysplastic hips of children are varied and thicker compared to those in adults.

The 3-D determination of the resultant joint force presented in this study is a simple but efficient expansion of the Ninomiya's method which is derived from the 2D static equilibrium equations using frontal X-ray. The direction of the abductor force, $\boldsymbol{F}$, was defined from the muscle attachment points in frontal and sagittal planes. Then, the resultant joint force, $\boldsymbol{R}$, was derived from the 3-D static equilibrium equations.

The results showed that the shelf operation changed the maximum contact pressures and the contact area as well as the anatomical parameters. The anatomical parameters after surgery shifted to the normal population of adult hips, which provide an indirect and qualitative sign of clinical outcomes. In addition, these anatomical parameters measured from the finite element models have good agreements with those measured in X-ray images postoperatively. The contact pressures and the contact area are reduced after surgery, and these mechanical parameters provide the direct guideline of the optimal distraction length $\left(\boldsymbol{l}_{d}\right)$ of the shelf operation. The ratio of the contact area over $60 \%$ of the maximum contact pressure could be another biomechanical guideline for surgical planning. This ratio represents how the contact pressures are well 
distributed. So, in the planning of the shelf operation, both the maximum contact pressure and the ratio of the contact area over $60 \%$ of the maximum contact pressure should be considered to minimize.

There are several assumptions and limitations in this study. First, the cartilage thicknesses on femoral and acetabular sides are approximately estimated from the data points of frontal and sagittal MR images. However this approximation may not change the thickness values dramatically since the response surface could well approximate the real cartilage surface within appropriate polynomial orders. The material properties of both the bones and the cartilage are assumed as linear elastic referred to the literature ${ }^{(9)}$. In addition, the material properties of the bones are assumed constant, but this assumption will not change the analysis results much since most of the deformations occurred in the cartilage of the contact surface. We only considered a standing posture, and it would be necessary to apply the realistic resultant joint forces in gait cycle. Also, different boundary conditions may change the results.

\section{Conclusion}

CT and MR image based finite element contact analyses of two dysplastic hips were conducted in the preoperative and post-operative shelf operation. The cartilage thickness was approximately measured from MR images. A new method to estimate the joint contact force based on the 3-D expansion of the Ninomiya's method using the 3-D FE models. The surgical planning based on clinical guidelines could improve both anatomical parameters and mechanical parameters. Major anatomical parameters shifted to the normal range of adult hips. The contact pressures decreased while the contact area increased after surgery. To obtain the optimal surgical planning, both maximum contact pressure and pressure distribution should be considered to minimize. The present method could be clinically useful for the optimal planning of the shelf operation in hip dysplasia.

\section{Acknowledgement}

This research was supported by the Kyung Hee University Research Fund in 2005 (KHU-20050310), Korea.

\section{References}

( 1 ) Kruse, R.W., Guille, J.T. and Bowen, J.R., Shelf Arthroplasty in Patients Who Have Legg-CalvePerthes Disease, J. Bone Joint Surg. Am., Vol.73-A, No.9 (1991), pp.1338-1347.

(2 ) Nishimatsu, H., Iida, H., Kawanabe, K., Tamura, J. and Nakamura, T., The Modified Spitzy Shelf Operation for Patients with Dysplasia of the Hip, J. Bone Joint Surg.
Br., Vol.84-B, No.5 (2002), pp.647-652.

( 3 ) Jacobs, R., Moens, P. and Fabry, G., Lateral Shelf Acetabuloplasty in the Early Stage of Legg-CalvePerthes Disease with Special Emphasis on the Remaining Growth of the Acetabulum: A Preliminary Report, J. Pediatric Ortho. B, Vol.13, No.1 (2004), pp.21-28.

(4) Wainwright, D., The Shelf Operation for Hip Dysplasia in Adolescence, J. Bone Joint Surg. Br., Vol.58, No.2 (1976), pp.159-163.

( 5 ) Ninomiya, S., Tagawa, H., Miyanaga, Y. and Seki, N., The Relationship between the Position of the Artificial Joint and the Resultant Force Acting on the Femoral Head, J. Jpn. Orthop. Ass., Vol.50, No.1 (1975), pp.1520.

(6) Genda, E., Iwasaki, N., Li, G., MacWilliams, B.A., Barrance, P.J. and Chao, Y.S., Normal Hip Joint Contact Pressure Distribution in Single-Leg Standing-Effect of Gender and Anatomic Parameters, J. Biomech., Vol.34, No.7 (2001), pp.895-905.

( 7 ) Pompe, B., Daniel, M., Sochor, M., Vengust, R. and Kralj-Iglic, V., Gradient of Contact Stress in Normal and Dysplastic Human Hips, Med. Eng. Phys., Vol.25, No.5 (2003), pp.379-385.

( 8 ) Mavcic, B., Pompe, B., Antolic, V., Daniel, M., Iglic, A. and Kralj-Iglic, V., Mathematical Estimation of Stress Distribution in Normal and Dysplastic Human Hips, J. Ortho. Res., Vol.20, No.5 (2002), pp.10251030.

( 9 ) Wei, H.W., Sun, S.S., Jao, S.H., Yeh, C.R. and Cheng, C.K., The Influence of Mechanical Properties of Subchondral Plate, Femoral Head and Neck on Dynamic Stress Distribution of the Articular Cartilage, Med. Eng. Phys., Vol.27, No.4 (2005), pp.295-304.

(10) Spoor, C.W., Van Leeuwen, L.J., De Windt, F.H.J. and Huson, A., A Model Study of Muscle Forces and JointForce Direction in Normal and Dysplastic Neonatal Hips, J. Biomech., Vol.22, No.8/9 (1989), pp.873-884.

(11) Wu, Y.T., From CT Image to 3D Model, Advanced Imaging, Vol.16, No.8 (2001), pp.20-23.

(12) Pal, N.R. and Pal, S.K., A Review on Image Segmentation Techniques, Pattern Recognition, Vol.26, No.9 (1993), pp.1277-1294.

(13) Saito, S., Takaoka, K. and Ono, K., Tectoplasty for Painful Dislocation or Subluxation of the Hip. LongTerm Evaluation of a New Acetabuloplasty, J. Bone Joint Surg. Br., Vol.68, No.1 (1986), pp.55-60.

(14) Nishii, T., Sugano, N., Sato, Y., Tanaka, H., Miki, H. and Yoshikawa, H., Three-Dimensional Distribution of Acetabular Cartilage Thickness in Patients with Hip Dysplasia: A Fully Automated Computational Analysis of MR Imaging, Osteoarth. Cartil., Vol.12, No.8 (2004), pp.650-657.

(15) Bartos, M. and Kestranek, Z., Numerical Solution of the Contact Problem. Application to a Simple Model of the Human Hip Joint, J. Comput. Appl. Math., Vol.63, No.1-3 (1995), pp.439-447. 